\title{
Examining the Association between Community-Level Marginalization and Emergency Room Wait Time in Ontario, Canada
}

\author{
Examen du lien entre la marginalisation \\ communautaire et le temps d'attente au service des \\ urgences en Ontario, Canada
}

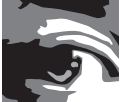 \\ ERICA J. MCDONALD, BSc, MSc (C) \\ School of Public Health and Health Systems \\ University of Waterloo \\ Waterloo, ON \\ MATTHEW QUICK, MES, PHD \\ Assistant Professor \\ School of Geographical Sciences E Urban Planning \\ Arizona State University \\ Tempe, $A Z$ \\ MARK OREMUS, MSc, PHD \\ Associate Professor \\ School of Public Health and Health Systems \\ University of Waterloo \\ Waterloo, ON
}

Abstract

Objective: This study examines the association between community-level marginalization and emergency room (ER) wait time in Ontario.

Methods: Data sources included ER wait time data and Ontario Marginalization Index scores. Linear regression models were used to quantify the association.

Results: A positive association between total marginalization and overall, high-acuity and low-acuity ER wait time was found. Considering specific marginalization dimensions, we found positive associations between residential instability and ER wait time and negative associations between dependency and ER wait time. 
Examining the Association between Community-Level Marginalization and

Emergency Room Wait Time in Ontario, Canada

Conclusions: Reductions in community-level marginalization may impact ER wait time. Future studies using individual-level data are necessary.

\section{Résumé}

Objectif: Cette étude examine le lien entre la marginalisation communautaire et le temps d'attente au service des urgences en Ontario.

Méthode: Les sources consultées comprenaient les données sur les temps d’attente aux urgences ainsi que les données de l'indice de marginalisation ontarien. La quantification des liens séest faite au moyen des modèles de régression linéaire.

Résultats : Nous avons constaté un lien positif entre une marginalisation totale et le temps d'attente aux urgences pour les affections générales, complexes et mineures. Quant aux aspects propres à la marginalisation, nous avons observé des liens positifs entre l'instabilité résidentielle et le temps d'attente aux urgences, et des liens négatifs entre la toxicomanie et le temps d'attente aux urgences.

Conclusion: Une réduction de la marginalisation communautaire pourrait avoir une incidence sur les temps d'attente aux urgences. Il faudrait maintenant mener des études qui se pencheraient sur les données au niveau individuel.

\section{Introduction}

The emergency room (ER) is a major point of entry for individuals requiring healthcare. Across developed countries, timely access to ER care is a priority, and high ER wait time reflects an inability of healthcare systems to adequately meet the needs of their populations (Downing et al. 2004; Watson et al. 2007). For individuals using the ER to access care, the impact of long wait times is profound; increased wait times are associated with decreased satisfaction and quality of care and increased risk of physical discomfort, medical error, morbidity and in-hospital mortality (Giuntella et al. 2018; Health Quality Ontario 2016; Mahmoud and Hou 2012; Marco et al. 2012; Vegting et al. 2015). Past studies have shown that hospital size, visit frequency and the acuity of visits explain some, but not all, of the variations in ER wait time between hospitals (Horwitz et al. 2010; Knowles et al. 2017). Typically, low-acuity (non-emergent) ER visits contribute less to ER wait time per visit and in total (Dinh et al. 2016); in contrast, high-acuity (emergent) visits preceding hospital admission have the longest ER wait time (Horwitz et al. 2010). Two studies defined ER wait time: Giuntella et al. (2018) defined it as the time from triage until discharge or hospital admission; Horwitz et al. (2010) defined it as the time from triage until first assessment by a physician.

Despite the importance of ER wait time as an indicator of healthcare access, no studies have, to the best of the authors' knowledge, considered how ER wait time may be higher or lower across communities with different levels of marginalization. Marginalization is 
defined as material and social disadvantage in one social group compared to other social groups. Marginalization can be evidenced by low socio-economic status, residential instability, poverty, dependency and poor healthcare utilization (Lynam and Cowley 2007). Marginalization impacts mental and physical health across the life course (Lynam and Cowley 2007).

Past research on the links between marginalization and the healthcare system focused on the health system in the US and Europe. These investigations have primarily examined the number of ER visits, rather than ER wait time, and have shown that marginalized groups visit ERs more frequently and require greater levels of care than non-marginalized groups. For example, marginalized people who are homeless in the US are less likely to use primary and preventive care, potentially increasing the number of ER visits in areas with relatively large homeless populations (Kushel et al. 2006). Likewise, marginalized individuals with chronic conditions are more likely than their non-marginalized counterparts to delay care, possibly resulting in a greater need for intensive ER services and higher visit frequencies in European and US ERs (Begley et al. 2011; Gunnarsson et al. 2013). Notwithstanding delayed care, economically disadvantaged persons in the US are more likely to present to ERs with poorer overall health than individuals from higher socio-economic strata (Bisgaier and Rhodes 2011).

Research exploring possible associations between marginalization and ER service use is inconclusive, and no past research has, to the authors' knowledge, examined the association between marginalization and ER wait time. Some work suggests that poor access to primary care physicians, which is more common among marginalized populations, may be associated with larger numbers of ER visits (Harris et al. 2011). However, research focused on Canadian ERs highlights that factors such as low educational attainment and low income at the individual and community levels may be more important determinants of high levels of ER utilization than poor access to physicians (Mian and Pong 2012; Ohle et al. 2017). Within the UK, greater community deprivation is associated with increased numbers of low-acuity ER visits (Harris et al. 2011). These findings contrast with studies suggesting that marginalized groups in the US use ER services less frequently (Tarraf et al. 2015).

Given the paucity of research exploring ER wait time and marginalization, we searched the literature for all developed countries and could not find any published research exploring whether ER wait time varies between communities with different levels of marginalization. Additionally, we found limited Canadian research that explores how community-level marginalization impacts ER care and no information about marginalization and ER wait time. As a result, we examined the association between community-level marginalization and ER wait time in the province of Ontario, Canada. Specifically, we looked at total communitylevel marginalization and four specific dimensions of marginalization, namely, residential instability, material deprivation, dependency and ethnic concentration.

This study reflects a principal goal of public health, namely, to facilitate equitable access to healthcare (Taillepierre et al. 2016). One important means of fostering equity is to focus 
Examining the Association between Community-Level Marginalization and Emergency Room Wait Time in Ontario, Canada

on the social determinants of health, including marginalization (Hall et al. 2016). The results of this study will be valuable for policy makers, health system managers and politicians because the study will serve as the first step toward understanding the degree to which community-level marginalization is associated with ER care in the form of wait time.

\section{Data and Methods}

\section{Study region and data}

Ontario is Canada's most populous province, with over 14 million inhabitants and approximately 39\% of the country's total population. In 2017, 166 Ontario hospitals had an ER and, as part of the Ontario Wait Time Strategy (Ontario Ministry of Health and Long-Term Care 2013), the Ontario Government provided publicly available wait time information for 119 of these ERs. We obtained the 2017 ER wait time data from the provincial government in fall 2018, before these data were removed from public access. ER wait times were measured in hours and calculated as the length of time between triage (i.e., being seen by a nurse upon arrival) and discharge from the ER, either to be sent home or admitted to the hospital.

The ER wait time data included measures of the median, average and 90th percentile wait time for three types of ER visits: all ER visits, high-acuity ER visits and low-acuity ER visits. Acuity ratings were based on ER nurses' triaging of new arrivals to the ER, with triaging undertaken using the Canadian Triage and Acuity Scale (CTAS). CTAS scores ranged from 1 (most serious) to 5 (least serious); scores between 1 and 3 indicated highacuity patients and scores between 4 and 5 indicated low-acuity patients (Health Quality Ontario 2018).

Marginalization data were obtained from the 2016 version of the Ontario Marginalization Index (ON-Marg) at the census subdivision level (CSD) (Public Health Ontario 2016a). CSDs approximate the geographical boundaries of municipalities (Statistics Canada 2018). Of the 575 CSDs in Ontario, 411 had marginalization data available through ON-Marg (Public Health Ontario 2016a). ON-Marg uses census data to measure total marginalization and four specific dimensions of marginalization: residential instability (type and density of residential accommodations and family structures), material deprivation (income, education, housing quality and family structures), dependency (number of children, seniors and those not participating in work) and ethnic concentration (visible minority status) (Public Health Ontario 2016a). These four dimensions are independent of one another and provide a more comprehensive representation of marginalization than other area-based deprivation indices that focus only on low socio-economic status (Matheson et al. 2012). The ON-Marg scores were calculated using 18 census variables and converted to standardized scores $(\mu=0, \sigma=1)$. High index scores indicated higher levels of marginalization. Further details about ON-Marg are available elsewhere (Public Health Ontario 2016b). 


\section{Aligning hospital and census data}

Because ER wait time data were available at the hospital level and marginalization data were available at the CSD level, our analysis had to account for the geographical misalignment of these data. To do so, we first geocoded the street addresses of the 166 Ontario ERs (Figure 1a) to the geographical coordinates (latitudes and longitudes). Second, we created a set of Thiessen polygons (Kopec 1963) to define the service area for each of the ERs (Figure 1b). Thiessen polygons take a set of input points (i.e., hospital locations) and construct one polygon around each input point such that any location within the polygon is closest to only its input point and not any other input point in the data set (Brassel and Reif 1979). Applied to define ER service areas, Thiessen polygons assume that individuals living within a given service area are more likely to visit the nearest hospital than any other hospital in the province. For reference, Thiessen polygons are often used in contexts where the "closest assignment" is considered reasonable, such as in the creation of hospital service areas, school service areas and coverage areas for police, fire and emergency services (Feng and Murray 2018; Pearce 2000; Schuurman et al. 2006).

FIGURE 1. ER service areas for the province of Ontario

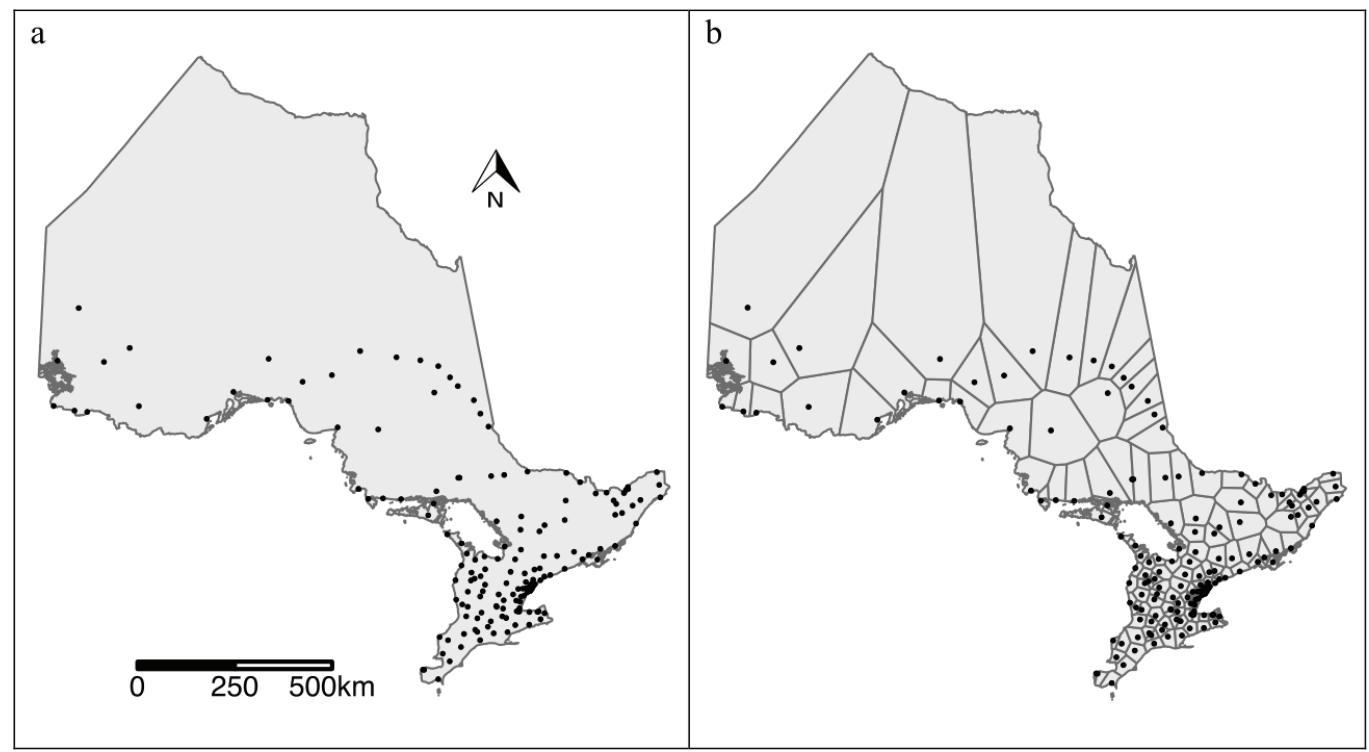

(a) Locations of ERs with available wait time data in the province of Ontario $(n=166)$ and (b) Service area boundaries defined using Thiessen polygons

\section{Calculating marginalization scores for ER service areas}

Each CSD was assigned to one or more ER service areas based on the intersection of the CSD boundaries and the ER service area boundaries. Marginalization scores were calculated for each ER service area using three approaches: arithmetic mean, geographically weighted average and population-weighted average. The arithmetic mean approach calculates service area marginalization scores as the average of the marginalization scores of the assigned 
CSDs. This assumes that each CSD that intersects with a service area has an equal weight (or contribution) to the marginalization score within a service area but does not consider differences in CSD size or population. For the geographically weighted average, service area marginalization scores were calculated based on the proportion of CSD area $\left(\mathrm{km}^{2}\right)$ that overlapped with a service area. For example, if two CSDs overlapped with one ER service area and constituted $90 \%$ and $10 \%$ of the total service area size, then the geographical weights used to calculate service area marginalization were 0.9 and 0.1 , respectively. For the population-weighted average, we used population weights based on the relative population sizes of the overlapping CSDs to calculate service area marginalization scores. In this case, CSDs with large population sizes had larger contributions to the service area marginalization scores than CSDs with small population sizes. Comparing and contrasting the results of the average, geographically weighted and population-weighted marginalization scores helps to understand the degree to which these assumptions influence the association between ER wait times and community-level marginalization.

Because some CSDs had missing marginalization data, when less than 50\% of the CSDs assigned to a given service area had missing data, the total marginalization scores for the ER service area were calculated as the average of the available CSD marginalization scores. When more than $50 \%$ of the CSDs assigned to a given service area had missing marginalization data, a marginalization score was not assigned to the ER service area and it was excluded from analysis. Missingness of $50 \%$ or more was used as the cut-off because any resulting average marginalization scores would not represent a majority of an ER service area's population.

\section{Regression analysis}

The associations between marginalization and ER wait time were quantified via three sets of linear regression models. Each set of models focused on a different method of computing ER wait time: median wait time, average wait time and 90th percentile wait time. Within each set of models, we ran subsets of models based on wait times for overall, low-acuity and highacuity ER visits. We examined different acuity levels because of reports that marginalized populations were more likely to visit ERs for low-acuity concerns (Mahmoud and Hou 2012; VanStone et al. 2013). As such, areas with high marginalization could have longer wait times for low-acuity visits than all ER visits or high-acuity visits. Each visit type was regressed on total marginalization and the four specific dimensions of marginalization in separate regression models. The modelling approach is depicted in Figure 2. We re-ran each set of models three times to account for the different weighting schemes used to compute service area marginalization scores (arithmetic mean, geographically weighted and population-weighted).

\section{Software and ethics}

We used QGIS v3.4.1 mapping software (Geographic Information System - Open Source Geospatial Foundation Project, Geneva, Switzerland) to create the ER service areas and 
FIGURE 2. Data analysis approach used to quantify the association between ER wait time and marginalization

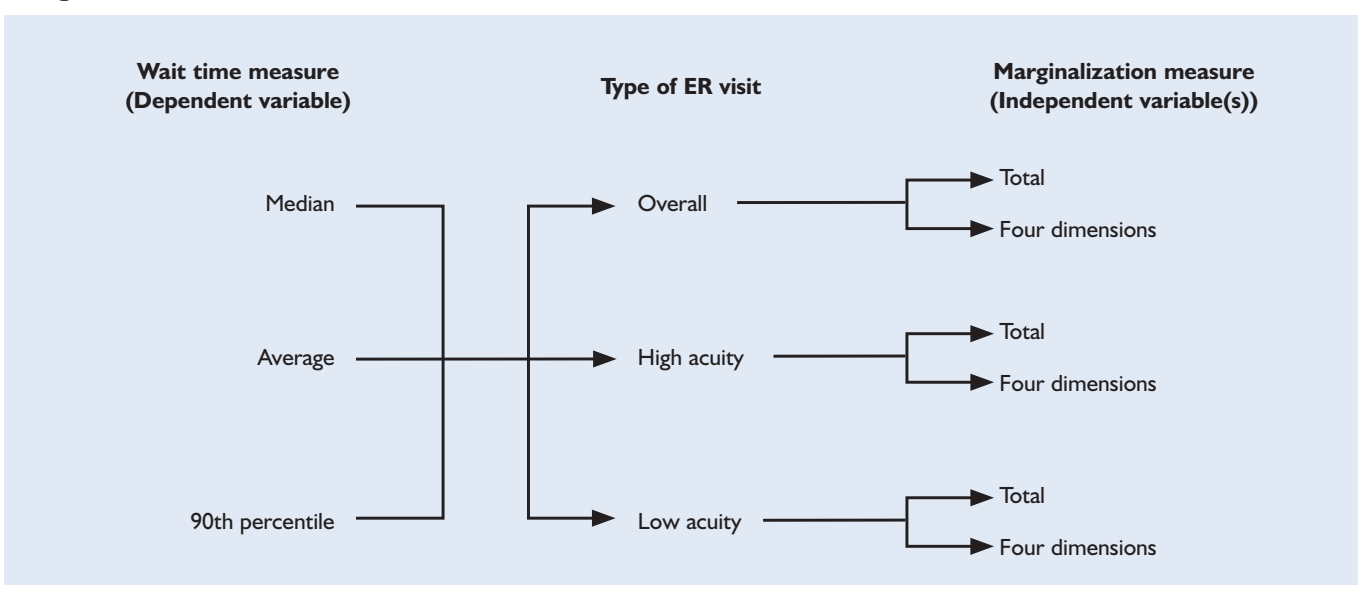

assign the CSDs to the service areas. The regression analyses were undertaken using R v3.5.1 (R Foundation for Statistical Computing, Vienna, Austria). The threshold of statistical significance was $\alpha=0.05$.

This study did not require research ethics approval because it was conducted with publicly available data collected at the population level.

\section{Results}

Overall, two types of data missingness were possible. The first occurred when more than $50 \%$ of CSDs had missing data, as a marginalization score was not assigned to the ER service area. The second type of missingness occurred when ER wait time data were missing. Of the 166 service areas created, 118 were included in our analyses because these had both ER wait time and marginalization data. There were 33 ER service areas that had missing marginalization data in over $50 \%$ of the CSDs assigned; these areas were not included in our analyses. In addition, there were 15 ER service areas that had marginalization scores assigned to the service area but were missing ER wait time data; these ER service areas were also not included in our analyses.

Using the Wilcoxon rank-sum test, we found some statistically significant differences in marginalization scores between the ER service areas with $(n=118)$ and without $(n=$ 15) missing ER wait time data and with less than 50\% of CSDs missing marginalization data, specifically among the dimensions of dependency, deprivation and ethnic concentration (Tables 1-3, available online at www.longwoods.com/content/26223). There were no statistically significant differences in marginalization scores between ER service areas with $(n=118)$ and without $(n=15)$ missing ER wait time data and with less than $50 \%$ missing marginalization data for total marginalization and residential instability.

In general, the coefficient signs, magnitudes and statistical significance levels were relatively consistent across the regression models analyzing median ER wait times and the three 
Examining the Association between Community-Level Marginalization and

Emergency Room Wait Time in Ontario, Canada

different versions of marginalization (Figure 3). Focusing on total marginalization, a positive and statistically significant association with overall and low-acuity median ER wait time was observed for the arithmetic mean, geographically weighted and population-weighted versions of marginalization (Tables 4-6, available online at www.longwoods.com/content/26223). Total marginalization was also positively associated with median ER wait time for highacuity visits; however, this coefficient was not statistically significant.

FIGURE 3. Regression coefficients and $95 \%$ confidence intervals for median ER wait time and marginalization
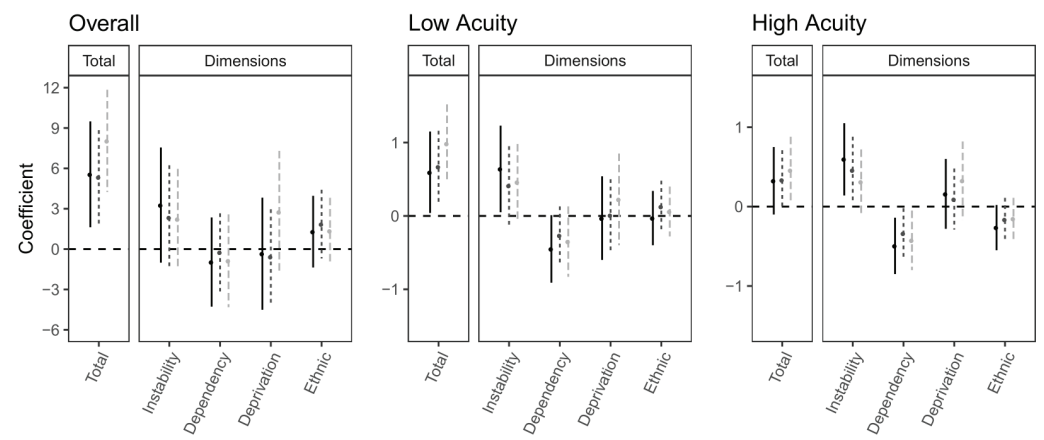

Marginalization

- Arithmetic mean

-... Geographically weighted average

-... Population-weighted average

Considering the individual dimensions of marginalization, residential instability was positively associated with median ER wait time for overall, high-acuity and low-acuity visits across all marginalization calculations; however, this association was only statistically significant for the arithmetic mean (low and high acuity) and geographically weighted (high acuity) versions of marginalization. Furthermore, there was a negative association between dependency and overall, high-acuity and low-acuity median ER wait time that was observed consistently across the arithmetic mean, geographically weighted and population-weighted versions of marginalization. Like residential instability, however, these results had varying levels of statistical (in)significance. There was no clear relationship with median ER wait time for all types of visits when considering the deprivation and ethnic concentration dimensions of marginalization. In the multiple regression models, the variance inflation factors were less than five, which suggested that multicollinearity did not impact the model results.

Consistent with the results for median ER wait time, statistically significant positive associations were observed between total marginalization and both overall wait times and low-acuity wait times for the average and 90th percentile wait time measures (Tables 7-12, available online at www.longwoods.com/content/26223). Further, our results exploring the association with instability and ER wait time were consistent across all wait time variables and observed across all marginalization calculations (Figures 4 and 5, available online at www.longwoods.com/content/26223). 


\section{Discussion}

To our knowledge, the present study is the first to consider marginalization for ER service areas in Ontario or elsewhere. Further, this is the only study to examine ER wait time, rather than Ontario ER visit frequency (VanStone et al. 2013), as the outcome in relation to community marginalization. We found population-level positive associations between total marginalization and median ER wait time in Ontario.

\section{Interpretation}

Our results suggest that communities with higher levels of total marginalization, and residential instability in particular, have longer ER wait times across all types of visits as well as both high- and low-acuity visits.

Our results complement the findings of social determinants of health research, which often report associations between high levels of marginalization and lower access to healthcare. Previous studies show positive associations between greater community deprivation and increased numbers of low-acuity ER visits (Harris et al. 2011). Other studies link minority status, material disadvantage and economic disadvantage to increased use of ER services (Haggerty et al. 2007; Kushel et al. 2006; Mian and Pong 2012). Therefore, our findings appear to be situated in a chain of events, whereby marginalization increases the frequency of ER visits, which places a heavier demand on ER services and leads to a longer ER wait time.

We found that instability as a specific dimension of marginalization was positively associated with ER wait times. This result aligns with investigations showing that residential instability is a crucial social determinant of health (Carder et al. 2018; Gadermann et al. 2019). Within a Canadian context, recent studies have linked residential instability to poor health status and increased healthcare utilization (Harris et al. 2019; Jaworsky et al. 2016). Aspects of residential instability, such as living alone or having recently moved, may result in an increased need for emergency services whereby individuals are brought to ER settings. Within the context of our research, increased healthcare utilization may place a greater demand on ER services, increasing ER wait time overall.

To date, to our knowledge, no studies have considered community levels of dependency, deprivation or ethnic concentration and their associations with ER wait time. We found levels of dependency, defined by ON-Marg as the number of children, seniors and adults who are not participating in paid work, to be inversely associated with ER wait time. These results appear to counter published studies undertaken in related research areas. For example, at the individual level, unemployment was found to be positively associated with healthcare utilization in Canada (after controlling for mental health treatment) (Kraut et al. 2000) and the US (Kuka 2000). One would thus expect to find longer wait times in areas with higher levels of dependency. Perhaps our results reflect the ecological fallacy, and further research using individual-level data is required to explain our findings.

The results for deprivation and ethnic concentration do not show any stable patterns across the different analyses. At the community level, this suggests an absence of effect for 
these two dimensions of marginalization. However, individual-level relations may differ from the findings of our study.

The results from our study can inform the policy process. Sound public health initiatives should be based on evidence suggesting the potential for change (Baicker and Chandra 2017; Roberge et al. 2010). In the context of the social determinants of health, the types of initiatives that bring about positive changes to the social condition and improve population health are often resource intensive. Prior to the initiation of new policies, evidence-based assessments should be completed to assess whether the potential health benefits will eclipse the cost of resources. The reduction of community-level marginalization is a resource-intensive process; however, our study suggests that this process may reduce ER wait time, particularly if efforts are focused on reducing community residential instability. Policy makers can use this information to further evaluate the health and social benefits, and costs, of programs designed to ameliorate community-level marginalization.

\section{Strengths and limitations}

One strength of this study was the ON-Marg data set's use of large sample sizes to generate marginalization scores. In addition, ON-Marg is stable across diverse geographical areas, such as urban and rural areas (Public Health Ontario 2016b). Marginalization scores assigned to CSDs were likely to be representative of the levels and types of marginalization experienced by Ontario communities (Public Health Ontario 2016b). Of course, our study used ecological-level data, meaning that its results might not be applicable at the individual level.

Another notable strength of this work is the robust analyses completed. By using three marginalization calculations (arithmetic mean, geographically weighted and population-weighted), we complete the same analyses with different assumptions regarding the aggregation of data across spatial scales. Similarly, median average and 90th percentile wait time measures were considered. Comparing the results of these analyses shows that there is consistency in our results, providing evidence that the results of this study reflect an ecological association between community marginalization and ER wait time.

One limitation of this study is that the Thiessen polygon method for developing ER service areas assumes that individuals attend the ER closest to their place of residence. This assumption may not always be true and could have introduced non-differential misclassification into our study, thereby biasing results toward the null hypothesis.

Moreover, our analyses had to overcome missing data. Although we could not comment on the nature of the missingness, we acknowledge that marginalization was, at times, greater for service areas with missing wait time data than for those without missing wait time data. If complete marginalization data were available, the strength of the inverse associations seen for dependency would decrease, and the strength of the positive association seen for deprivation would increase. With respect to the ethnic concentration dimension, areas with missing ER wait times had lower marginalization scores than areas with complete wait time 
data. Complete data for this dimension would result in the positive associations being pulled toward the null and inverse associations moving away from the null. Overall, compared to residential instability and total marginalization, less confidence can be placed in the results for the dependency, deprivation and ethnic concentration dimensions (Tables 1-12 and Figures 4 and 5, available online at www.longwoods.com/content/26223).

Finally, several potentially useful covariates were not publicly available. Hospital capacity and overcrowding may contribute to ER wait time (Horwitz et al. 2010; Knowles et al. 2017), yet the provincial government did not make these data publicly available. Data on time to first assessment by a physician were also not publicly available. Using a populationweighted approach to calculate marginalization scores allowed us to consider population as a surrogate for hospital capacity and visit frequency. Future work should incorporate measures of hospital capacity and time to first assessment by a physician in regression models.

In conclusion, this study shows that total community-level marginalization is associated with increased ER wait time in Ontario, particularly for the residential instability dimension of marginalization. Further investigation of this association at the individual level is necessary to gain a more complete understanding of the link between marginalization and ER wait time.

\section{Acknowledgements}

Joint acknowledgement/disclosure statement: This project was undertaken without funding.

Correspondence may be directed to: Mark Oremus, School of Public Health and Health Systems, University of Waterloo, 200 University Avenue, Waterloo, ON N2L 3G1. He can be reached by phone at 519-888-4567x35129 or by e-mail at moremus@uwaterloo.ca.

\section{References}

Baicker, K. and A. Chandra. 2017. Evidence-Based Health Policy. NEJM 377: 2413-15. doi:10.1056/ NEJMp1709816.

Begley, C.E., R. Basu, D. Lairson, T. Reynolds, S. Dubinsky, M. Newmark et al. 2011. Socioeconomic Status, Health Care Use, and Outcomes: Persistence of Disparities over Time. Epilepsia 52(5): 95764. doi:10.1111/j.1528-1167.2010.02968.x.

Bisgaier, J. and K.V. Rhodes. 2011. Cumulative Adverse Financial Circumstances: Associations with Patient Health Status and Behaviors. Health \& Social Work 36(2): 129-37. doi:10.1093/hsw/36.2.129.

Brassel, K.E. and D. Reif. 1979. A Procedure to Generate Thiessen Polygons. Geographical Analysis 11(3): 289-303. doi:10.1111/j.1538-4632.1979.tb00695.x.

Carder, P.C., J. Kohon, A. Limburg and E. Becker. 2018. Waiting for Housing Assistance: Characteristics and Narrative Accounts of Low-Income Older Persons. Housing and Society 45(2): 63-80. doi:10.1080/08882746.2 018.1488212.

Dinh, M.M., S.B., Russell, K.J., Bein, D.R., Chalkley, D. Muscatello, R. Paoloni et al. 2016. Statewide Retrospective Study of Low Acuity Emergency Presentations in New South Wales, Australia: Who, What, Where and Why? BMJ Open 6(5): e010964. doi:10.1136/bmjopen-2015-010964.

Downing, A., R.C., Wilson and M.W., Cooke. 2004. Which Patients Spend More than 4 Hours in the Accident and Emergency Department? Journal of Public Health 26(2): 172-76. doi:10.1093/pubmed/fdh141. 


\section{Examining the Association between Community-Level Marginalization and Emergency Room Wait Time in Ontario, Canada}

Feng, X. and A.T. Murray. 2018. Allocation Using a Heterogeneous Space Voronoi Diagram. Journal of Geographical Systems 20(3): 207-26. doi:10.1007/s10109-018-0274-5.

Gadermann, A.M., M.E. Karim, M. Norena, S.D. Emerson, A.M. Hubley, L.B. Russell et al. 2019. The Association of Residential Instability and Hospitalizations among Homeless and Vulnerably Housed Individuals : Results from a Prospective Cohort Study. Journal of Urban Health 97(2): 239-49. doi:10.1007/ s11524-019-00406-9.

Giuntella, O., C. Nicodemo and C. Vargas-Silva. 2018. The Effects of Immigration on NHS Waiting Times. Journal of Health Economics 58: 123-43. doi.org/10.1016/j.jhealeco.2018.02.001.

Gunnarsson, H., A. Ekholm and L.I. Olsson. 2013. Emergency Presentation and Socioeconomic Status in Colon Cancer. European Journal of Surgical Oncology 39(8): 831-36. doi:10.1016/j.ejso.2013.04.004.

Haggerty, J.L., D. Roberge, R. Pineault, D. Larouche and N. Touati. 2007. Features of Primary Healthcare Clinics Associated with Patients' Utilization of Emergency Rooms: Urban-Rural Differences. Healthcare Policy 3(2): $72-85$.

Hall, M., C. Graffunder and M. Metzler. 2016. Policy Approaches to Advancing Health Equity. Journal of Public Health Management and Practice 22(Suppl 1): S50-59. doi:10.1097/PHH.0000000000000365.

Harris, M., A. Gadermann, M. Norena, M. To, A.M. Hubley, T. Aubry et al. 2019. Residential Moves and its Association with Substance Use, Healthcare Needs, and Acute Care Use among Homeless and Vulnerably Housed Persons in Canada. International Journal of Public Health 64(3): 399-409. doi:10.1007/ s00038-018-1167-6.

Harris, M.J., B. Patel and S. Bowen. 2011. Primary Care Access and Its Relationship with Emergency Department Utilisation: An Observational, Cross-Sectional, Ecological Study. British Journal of General Practice 61(593): 787-93. doi:10.3399/bjgp11X613124.

Health Quality Ontario. 2016. Under Pressure: Emergency Department Performance in Ontario. Retrieved July 30, 2019. <https://www.hqontario.ca/portals/0/Documents/system-performance/under-pressure-report-en.pdf >.

Health Quality Ontario. 2018. Health Quality Ontario Indicator Library. Retrieved October, 2018. <http:// indicatorlibrary.hqontario.ca/Indicator/Summary/Median-emergency-department-length-stay/EN>.

Horwitz, L., J. Green and E.H. Bradley. 2010. US Emergency Department Performance on Wait Time and Length of Visit. Annals of Emergency Medicine 55(2) 133-41. doi:10.1016/j.annemergmed.2009.07.023.

Jaworsky, D., A. Gadermann, A. Duhoux, T.E. Naismith, M. Norena, M.J. To et al. 2016. Residential Stability Reduces Unmet Health Care Needs and Emergency Department Utilization among a Cohort of Homeless and Vulnerably Housed Persons in Canada. Journal of Urban Health 93(4): 666-81. doi:10.1007/ s11524-016-0065-6.

Knowles, E., S.M. Mason and C. Smith. 2017. Factors Associated with Exit Block and Impact on the Emergency Department. Emergency Medicine Journal 34(1): 61-62. doi:10.1136/emermed-2015-205202.

Kopec, R.J. 1963. An Alternative Method for the Construction of Thiessen Polygons. Professional Geographer 15(5): 24-26. doi:10.1111/j.0033-0124.1963.024_r.x.

Kraut, A., C. Mustard, R. Walld and R.B. Tate. 2000. Unemployment and Health Care Utilization. Scand J Work Environ Health 26(2): 169-77. doi:10.5271/sjweh.527.

Kuka, E. 2000. Quantifying the Benefits of Social Insurance: Unemployment Insurance and Health. NBER Working Paper Series (24766). doi:10.3386/w24766.

Kushel, M.B., R. Gupta, L. Gee and J.S. Haas. 2006. Housing Instability and Food Insecurity as Barriers to Health Care among Low-Income Americans. Journal of General Internal Medicine 21(1): 71-77. doi:10.1111/j.1525-1497.2005.00278.x.

Lynam, M.J. and S. Cowley. 2007. Understanding Marginalization as a Social Determinant of Health. Critical Public Health 17(2): 137-49. doi:10.1080/09581590601045907.

Mahmoud, I. and X. Hou. 2012. Immigrants and the Utilization of Hospital Emergency Departments. World Journal of Emergency Medicine 3(4): 245-50. doi:10.5847/wjem.j.issn.1920-8642.2012.04.001.

Marco, C.A., M. Weiner, S.L. Ream, D. Lumbrezer and D. Karanovic. 2012. Access to Care among Emergency 
Department Patients. Emergency Medicine Journal 29(1): 28-31. doi:10.1136/emj.2010.103077.

Matheson, F.I., J.R. Dunn, K.L. Smith, R. Moineddin and R.H. Glazier. 2012. Development of the Canadian Marginalization Index: A New Tool for the Study of Inequality. Canadian Journal of Public Health 103(8 Suppl 2): S12-16.

Mian, O. and R. Pong. 2012. Does Better Access to FPs Decrease the Likelihood of Emergency Department Use? Canadian Family Physician 58(11): e658-66.

Ohle, R., M. Ohle and J.J. Perry. 2017. Factors Associated with Choosing the Emergency Department as the Primary Access Point to Health Care: A Canadian Population Cross-Sectional Study. CJEM 19(4): 27176. doi:10.1017/cem.2016.350.

Ontario Ministry of Health and Long-Term Care. 2013. Hospital Locations and Classifications by LHINs. Retrieved July 30, 2019. <http://www.health.gov.on.ca/en/common/system/services/hosp/locations.aspx >.

Pearce, J. 2000. Techniques for Defining School Areas for Comparison with Census Data. Computers, Environment and Urban Systems 24(4): 283-303. doi:10.1016/S0198-9715(99)00045-9.

Public Health Ontario. 2016a. 2016 Ontario Marginalization Index - User Guide. Retrieved October 2018. $<$ https://www.publichealthontario.ca/-/media/documents/O/2017/on-marg-userguide.pdf $>$.

Public Health Ontario. 2016b. Ontario Marginalization Index (ON-Marg). Retrieved July 30, 2019. $<$ https://www.publichealthontario.ca/en/DataAndAnalytics/Pages/ON-Marg.aspx>.

Roberge, D., R. Pineault, D. Larouche and L.R. Poirier. 2010. The Continuing Saga of Emergency Room Overcrowding: Are We Aiming at the Right Target? Healthcare Policy 5(3): 27-39. doi:10.12927/ hcpol.2013.21637.

Schuurman, N., R.S. Fiedler, S.C. Grzybowski and D. Grund. 2006. Defining Rational Hospital Catchments for Non-Urban Areas Based on Travel-Time. International Journal of Health Geographics 5(1): 43. doi:10.1186/1476-072X-5-43.

Statistics Canada. 2018. Census Consolidated Subdivision (CCS). Retrieved July 30, 2019. <https://www150. statcan.gc.ca/n1/pub/92-195-x/2011001/geo/ccs-sru/ccs-sru-eng.htm>.

Taillepierre, J.C.D., L. Liburd, A. O’Connor, J. Valentine, K. Bouye, D.H. McCree et al. 2016. Toward Achieving Health Equity: Emerging Evidence and Program Practice. Journal of Public Health Management and Practice 22(Suppl 1): S43-S49. doi:10.1097/PHH.0000000000000375.

Tarraf, W., W. Vega and H. González. 2015. Emergency Department Services Use among Immigrant and Non-Immigrant Groups in the United States. Journal of Immigrant and Minority Health 16(4): 595606. doi:10.1007/s10903-013-9802-z.

VanStone, N., P. Belanger, K. Moore and J.M. Caudle. 2013. The Socioeconomic Composition of Low-Acuity Emergency Department Users in Ontario. Canadian Family Physician 60(4): 355-62.

Vegting, I.L., N. Alam, K. Ghanes, O. Jouini, F. Mulder, M. Vreeburg et al. 2015. What Are We Waiting for? Factors Influencing Completion Times in an Academic and Peripheral Emergency Department. Netherlands Journal of Medicine 73(7): 331-40.

Watson, D.E., M.L. Barer, H.M. Matkovich and M.L. Gagnon. 2007. Wait Time Benchmarks, Research Evidence and the Knowledge Translation Process. Healthcare Policy 2(3): 56-62. 